A NEW study of the behaviour of the tide within the lagoon of Venice, in itself not a revolutionary idea, promises to have far-reaching practical results in the Italian government's choice of the most suitable barrier to prevent flooding in Venice.

A. Goldmann, R. Rabagliati and P. Sguazzero of the IBM Scientific Centre in Venice in an article in the Rivista Italiana di Geofisica (2, 119; 1975) have computed the relation between the level of water outside the lagoon and the level at 14 points within the $50 \mathrm{~km}^{2}$ lagoon. The data were provided by hourly readings of water level at the 15 stations during 1972 and 1973. Using spectral analysis, the authors first investigated the transfer of energy from the open sea to every lagoon station at each of a large number of frequency bands. This showed that only in three frequency bands - the long-term, the diurnal and the semi-diurnal-was the transfer of energy significant. The authors then calculated the gain or loss in the amplitude of the diurnal and semi-diurnal waves-those in which the astronomical components are predominant-and the time it took waves of these two frequencies to reach each station (the phase).

In 1971 the lagoon was sounded in detail: the soundings revealed that $75 \%$ of the lagoon is shallow water or marsh less than $2 \mathrm{~m}$ deep, intersected by the deep channels which make up the other $25 \%$. When this information is combined with the tide data an interesting picture begins to emerge: on deep channels the semidiurnal waves, although not the diurnal waves, actually increase in amplitude after entering the lagoon (the gain at the Grand Canal station is $6 \%$ ); in shallow water on the other hand the waves lose amplitude, the semi-diurnal waves losing more amplitude than the diurnal ones. Propagation of waves is slower in shallow

\section{On the lagoon}

\section{from Gillian Boucher}

water and the waves of the diurnal tide are propagated more slowly than those of the semi-diurnal.

The detailed results, with the advantages of a long period of data collection, the almost contemporary sounding data, and sophisticated statistical techniques, provide a clearer picture of the lagoon than has ever been obtained before. One important use for them will be to show how the lagoon is changing by comparison with past and future results. But the results have already been used in another of the IBM proiects, very recently completed: a mathematical model of the entire lagoon. The model, calibrated by the new tide results and other information, can reproduce the behaviour of water at any point and the effects of man-made changes in the lagoon. One of the first problems the model will be called upon to solve is the effect upon the lagoon of the various types of barrier which could be erected at its three entrances.

One of the scourges of Venice is the tide which, though small by Atlantic standards, frequently causes flooding because the city is so lowlying. To save the city from the saltwater inundations the Italian government has invited tenders for the construction of a sluice at the entrances to the lagoon; companies interested in tendering must do so by June 30 this year. Of the two basic types of barrier, the moveable and the fixed, the moveable (for example Pirelli's project for an inflatable barrier) are clearly superior but very much more expensive. A fixed barrier completely shutting off the lagoon is out of the question-Venice relies on the tide to wash away industrial and domestic pollution, and to prevent circulation of water would be devastating to both humans and buildings. So the choice is between a moveable barrier and some kind of partial fixed barrier which will lessen the quantity of water entering sufficiently to prevent flooding. Inevitably a fixed barrier will also reduce circulation. The IBM model will enable companies and the government to decide whether a satisfactory compromise can be obtained between the needs to damp the tides and to control pollution.
Orgel (J. molec. Biol., 38, 367 and 380; 1968 ) that the primitive ribosome consisted primarily of RNA, with the proteins being added later to fulfil more sophisticated functions. More recently, the functional importance of RNA in the "modern" ribosome has been preached by a number of authors, most notably by Kurland (Ribosomes, 309, Cold Spring Harbor Monograph, 1974), and the purpose of this article is to review very briefly the present status of rRNA, with regard to both structure and function.

Little progress can be made nowadays in any nucleic acid problem without previous knowledge of the base sequences involved. In the $E$. coli ribosome, the sequence of the 5S RNA component has been known for many years (Brownlee et al., J. molec. Biol., 34,$379 ; 1968$ ), and the primary structure of the $16 \mathrm{~S}$ RNA is now almost fully established (Ehresmann et al., Nucleic Acids Res., 2, 265; 1975), although there is still a measure of disagreement as to the detailed sequences of some of the oligonucleotide degradation products (Magrum et al., Nature, 257, 423; 1975). Progress has also been made in the sequencing of the 23S RNA (Branlant et al., Biochimie, 57, 175: 1975). The primary sequences themselves give a strong hint that the RNA is more than just a structural skeleton within the ribosome; the 5S sequence has been strongly conserved throughout evolution (reviewed by Monier, Ribosomes, 141, Cold Spring Harbor Monograph, 1974), and comparison of the oligonucleotide catalogues from 16S RNA molecules from different bacteria shows a remarkable degree of homogeneity (Woese et al., Nature, 254, 83; 1975), again indicating that substantial regions of the sequence have been conserved. The obvious inference is that particular oligonucleotide sequences are required to fulfil specific ribosomal functions, and this has tempted several authors to put forward hypotheses involving complementary base-pairing between regions of rRNA and other nucleic acid components of the protein synthesising system (for example, van Knippenberg, Nucleic Acids Res., 2, 79; 1975). Although these ideas are attractive, it must be borne in mind that in molecules the size of rRNA, any given nucleotide sequence of up to six bases would be expected to appear on a statistical basis, and solid experimental evidence to support this type of hypothesis is unfortunately very difficult to obtain.

Evidence has however been forthcoming in two cases. The first of these concerns the proposal made by Shine and Dalgarno (Proc. natn. Acad. Sci. U.S.A., 71, 1342; 1974) that the $3^{\prime}$ terminal sequence of $16 \mathrm{~S}$ RNA is involved in mRNA recognition. Here, Steitz and Jakes (Proc. natn. Acad. Sci. U.S.A., 72, 4734; 1975) have been able to isolate a dissociable oligonucleotide complex consisting of the 3'-terminus of the 16S RNA and an RNA fragment from a protein initiator region of R17 phage RNA (see also News and Views, 260, 12; 1976). The two fragments contained a sevenbase complementary sequence. The second case concerns the 5S RNA, where several lines of evidence suggest that binding of tRNA to the $50 \mathrm{~S}$ ribosome involves a complementary base-pairing between the T $\psi \mathrm{CG}$ loop of the latter with 5S RNA (Erdmann et al., Biochem, biophys. Res. Commun., 54, 942; 1973). Here there is some conflicting evidence, as the relevant G-residues on the 5S RNA 